\title{
Two-Way Flow Coupling in Ice Crystal Icing Simulation
}

\author{
Jonathan Paul Connolly University of Oxford
}

Geoffrey Jones Rolls-Royce Plc

Alex Bucknell, Liam Parker, Matthew McGilvray, and David Gillespie University of Oxford

Benjamin Collier Rolls-Royce Plc

Citation: Connolly, J.P., Jones, G., Bucknell, A., Parker, L. et al., "Two-Way Flow Coupling in Ice Crystal Icing Simulation," SAE Technical Paper 2019-01-1966, 2019, doi:10.4271/2019-01-1966.

\section{Abstract}

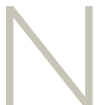

umerous turbofan power-loss events have occurred in high altitude locations in the presence of ice crystals. It is theorized that ice crystals enter the engine core, partially melt in the compressor and then accrete onto stator blade surfaces. This may lead to engine rollback, or shed induced blade damage, surge and/or flameout. The first generation of ice crystal icing predictive models use a single flow field where there is no accretion to calculate particle trajectories and accretion growth rates. Recent work completed at the University of Oxford has created an algorithm to automatically detect the edge of accretion from experimental video data. Using these accretion profiles, numerical simulations were carried out at discrete points in time using a manual meshing process. That work showed that flow field changes caused by a changing accretion profile had significant effects on the collection efficiency of impinging particles, ultimately affecting the mass of accreted ice and its shape. This paper discusses the development of the ICICLE numerical ice crystal icing code to include a fully automated two-way coupling between the accretion profile and flow field solution, to account for these effects. The numerical strategy; geometry redefinition, mesh update and flow field solution are discussed, followed by a comparison to experimental ice accretion of a simple 2D geometry and model predictions with and without flow field updating. The results showed that significant changes in leading edge accretion profiles were numerically predicted when the only the geometry was updated. Further changes then occurred when the flowfield was also updated.

\section{Introduction}

ngestion of high altitude ice crystals has been attributed to be the cause of numerous engine power-loss events. Many of these events, occurred at altitudes significantly in excess of $22,000 \mathrm{ft}$., the recognized upper limit of the existence of supercooled liquid water [1]. Ice crystal concentrations in excess of $2 \mathrm{~g} / \mathrm{m}^{3}$ occur in the updraft cores of tropical storms, particularly in the anvil region, which may extend up to around $60,000 \mathrm{ft}$. at the equator. It is theorized that ingestion of these particles into the warm engine core causes their sublimation, melting and evaporation, removing heat from surfaces and the airflow until refreezing can occur. Accretion is believed to occur on stationary surfaces - endwalls, intercompressor ducting and stators, causing blockage of the main gas path and loss of efficiency. This can lead to uncommanded loss of thrust (rollback), shedding of large accretions into downstream stages causing rotor blade damage, increased vibrations, surge and/or flameout. A 2005 review of data from over 60 known engine power-loss events on commercial aircraft since 1990 by the Engine Harmonization Working Group (EHWG) led to a new interim envelope for engine certification in glaciated and mixed phase conditions. Subsequently adopted by regulatory authorities, this has led to a rapid expansion of research effort in developing analytical and semi-empirical models for the phenomenon, as a method of demonstrating compliance with regulations.

As part of these efforts, a 2D ice crystal icing numerical model has been developed at the University of Oxford, which is referred to as the Ice Crystal Icing ComputationaL Environment (ICICLE). The model incorporates Lagrangian particle tracking, a two-way heat transfer and phase change model, bouncing, sticking and fragmentation models, and a thermodynamic accretion model based on an Extended Messinger Model which includes a three layer model for blade temperatures above $0^{\circ} \mathrm{C}$. Details of the complete model may be found in [2] and details of the thermodynamic accretion model in [3]. In the standard model, a flow field solution is imported and is unchanged throughout the simulation time (a 'frozen' flow field). The effect of the growing accretion on the flow field - and hence particle trajectories - is not accounted for. This simplification is also made in the first generation of ice crystal icing models - for example that of ONERA [4]], 
Wright et al [్] and Nilamdeen \& Habashi []. However, testing in ice crystal wind tunnels has shown accretion size after an exposure time of minutes may be of the same order of magnitude - or greater - as the characteristic dimension of the test article (such as the chord of a blade or diameter of an axisymmetric model) $[\underline{3}, \underline{7}]$. Recent testing of the Honeywell ALF502-R5 engine at NASA's PSL-3 altitude ice crystal test facility showed that blockage of stator passages in the LP booster stage of the compressor could reach $60 \%$ [9]. An appreciable alteration of the flow field and particle trajectories is therefore likely, which must be considered to accurately predict time-dependent growth rates.

Previous work at the University of Oxford analysed experimental accretion profiles from data collected at National Research Council of Canada's Research Altitude Test Facility RATFac [10]. By selecting profiles at a range of time intervals, meshes were manually created to produce a continuous phase numerical solution. Discrete phase trajectories were then calculated using the method by Bucknell [10] to determine the collection efficiency over the stator. The work showed that a large change in collection efficiency occurred when the accretion size got sufficiently large and indicated that numerical simulations should consider the effect of accretion growth on the surrounding flowfield. Figure 1 shows the results of this work, where the collection efficiency reduced during the simulation for all particle size distributions tested.

Various methods of mesh morphing have been applied in previous literature, and have been summarised by El-Batsh [11]. In the early work by ONERA, the accretion was represented as a deformation of the original mesh, and it was suggested that the deformed mesh could be reused in the icing code to perform multi-stepping ice growth [12], however the results of this method were not published. It has since been found that this method of mesh adaption is limited to relatively small accretions [13], which makes this method unsuitable for ice crystal icing in which excessive accretion can occur. ONERA have published recent data on the effect of flow field updating for supercooled liquid droplet accretion [14], and more recently applied it to mixed phase conditions [15]. However to date, the authors of this paper has not seen work focused on determining the effect of flow field updating for ice crystal icing.

FIGURE 1 Change in collection efficiency with ice exposure time for a range of particle size distributions (PSD). Definition of PSDs is shown in table 1.

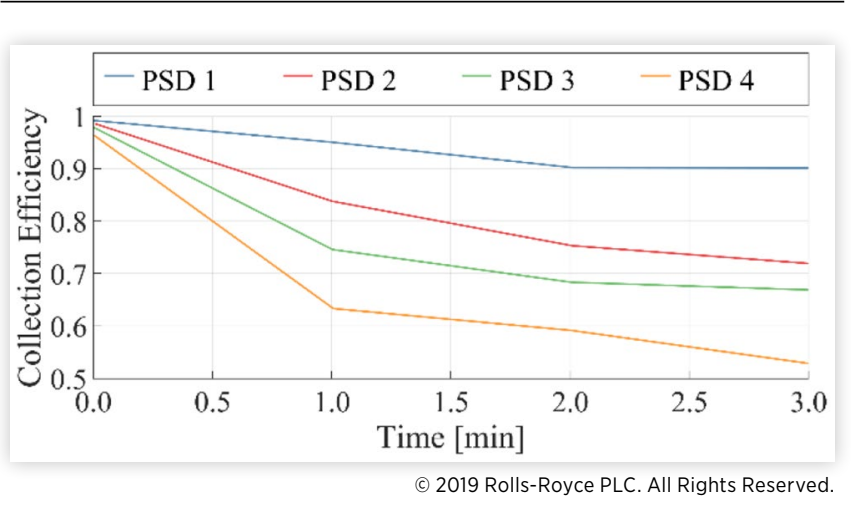

TABLE 1 Particle size distributions for the data shown in figure 1

\begin{tabular}{|l|l|l|l|}
\hline & $\mathbf{D}_{\mathrm{v} 10}(\boldsymbol{\mu m})$ & $\mathbf{D}_{\mathbf{v} 50}(\boldsymbol{\mu m})$ & $\mathbf{D}_{\mathrm{v} 90}(\boldsymbol{\mu m})$ \\
\hline PSD 1 & 18 & 34 & 54 \\
\hline PSD 2 & 28 & 40 & 80 \\
PSD 3 & 25 & 50 & 93 \\
\hline PSD 4 & 35 & 68 & 131 \\
\hline & & ( 2019 Rolls-Royce PLC. All Rights Reserved.
\end{tabular}

\section{ICICLE Overview}

A 2D ice crystal icing prediction code, named the Ice Crystal Icing ComputationaL Environment (ICICLE), has been created at the University of Oxford. In its current form, a two dimensional RANS simulation is computed using the geometry of the 'clean' test article (zero accretion). The flow field is then used within ICICLE for the Lagrangian particle tracking. Discrete streams of particles are injected into the domain at the inlet, with a Rosin-Rammler particle size distribution (which was deemed suitable to statistically represent the expected particle sizes present in the atmosphere). During the Lagrangian particle tracking, a two-way energy and mass coupling exists between the continuous and discrete phase (crystals).

Upon impact of the studied body, an energy based stick/ bounce/shatter model is applied to determine the post-impact behavior. Finally, a three-layer Extended Messenger Model is applied to determine the ice growth. The overall model is discussed in greater detail in [2], and the Messenger model details can be found in [8] .

An overview of the structure of the ICICLE code is shown in Figure 2. In its current form, time-stepping is achieved by injecting consecutive streams of particles and repeating the EMM calculations. This method uses the same flow-field for all time-steps. This paper will explain the extension to ICICLE, in which a continuous link between the predicted accretion shape and flow-field is produced. For the current work, every 15 seconds a new flow field is calculated and used within the ICICLE code.

FIGURE 2 Block diagram of the model. The blocks outlined in blue constitute the main time-stepping loop, grey represent input/output data, and orange being the new code described in this paper.

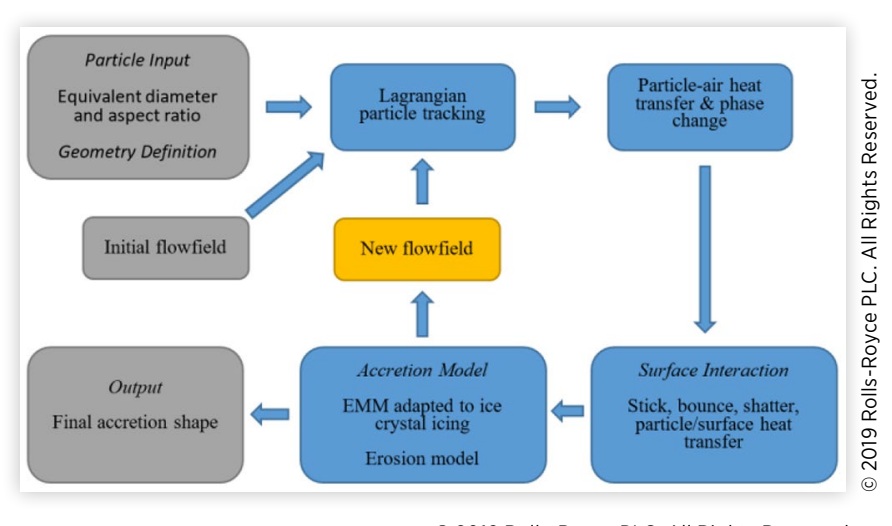

(c) 2019 Rolls-Royce PLC. All Rights Reserved. 


\section{Experimental Overview}

This paper studies the ice crystal icing on a prismatic stator blade, representative of a compressor blade (figure 3). The test article was experimentally studied at the National Research Council (NRC) of Canada's Research Altitude Test Facility (RATFac) in 2017. RATFac is an open loop, sub-atmospheric ice crystal icing tunnel which can be simplified to that shown in figure 4. A jet of ice and air is drawn into the tunnel by a downstream compressor. During the experiments, total and static pressure measurements were made upstream of the stator leading edge. Prior to starting the test, measurements of melt ratio (MR) were taken upstream of the stator leading edge, and measurements of the particle size distribution (PSD) were taken upstream of the tunnel bell-mouth.

This paper recreates the experiment which had an inlet total pressure of $34.5 \mathrm{kPa}$, inlet total temperature of $10^{\circ} \mathrm{C}$, freestream Mach number of 0.4 , relative humidity of $45 \%$ and a melt ratio of $5 \%, 50 \mathrm{~mm}$ upstream of the stator leading edge. The ice crystals were injected with $\mathrm{D}_{\mathrm{v} 10}, \mathrm{D}_{\mathrm{v} 50}, \mathrm{D}_{\mathrm{v} 90}$ of 18,34 , $54 \mu \mathrm{m}$ respectively. Full details of this experiment can be found in [10]. The numerical simulations discussed in this paper matched the experimentally measured boundary conditions. For the simulations detailed in this paper, the angle of attack equaled $0^{\circ}$. These conditions were chosen for this paper as it was at the "baseline" aerodynamic conditions and particle size distribution. Other experiments were carried out at the same aerodynamic conditions but a $22.5 \%$ relative humidity, and the numerical simulation of these tests showed good agreement with the experimental data [2], whereas at a higher relative humidity poorer performance was observed.

\section{Computational Domain}

To save on computational power, it was decided to truncate the domain into two sections; inlet and main domain (as shown in Figure 3). The flow developed within the inlet section, but due to a zero transverse pressure gradient the ice crystal trajectories were axial. Because of this, the simulation of the inlet section could be carried out once, and then successive simulations used the outlet conditions of this simulation as inlet conditions to the main CFD domain. The plane separating the inlet CFD domain and main CFD domain was chosen such that the particles at that point had on average deviated by one half of a cell size in the

\section{FIGURE 3 Isometric view of stator test article [9]}

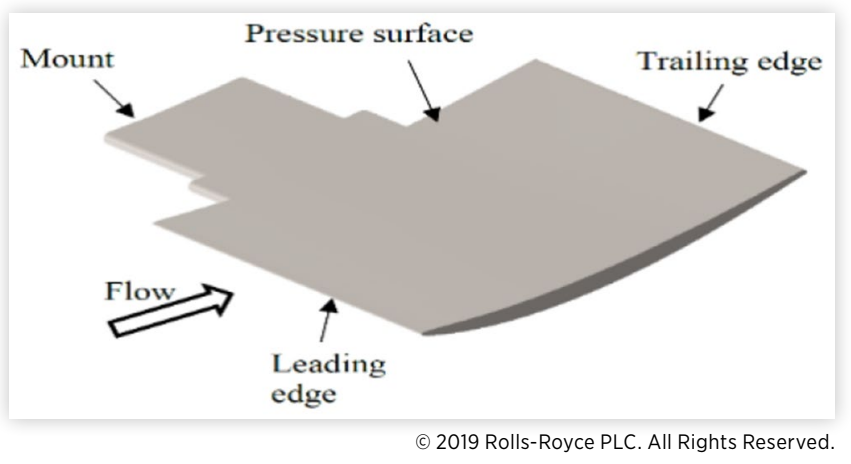

\section{FIGURE 4 Computational domain studied (not to scale)}

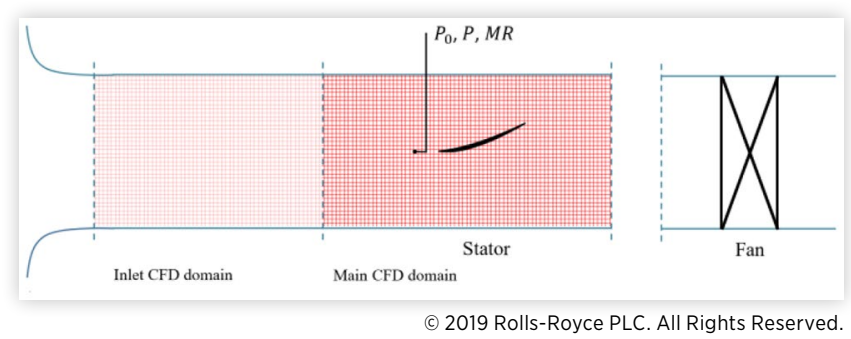

transverse direction relative to their original injection location. All particle properties (such as diameter, aspect ratio, melt ratio, temperature etc.) were matched between the outlet of the inlet CFD domain and inlet of the main CFD domain. The aerodynamic conditions were set at the start of the experiment by adjusting the outlet static pressure induced by the downstream fan. During the experiment, the fan settings were not changed, meaning that the aerodynamic conditions changed over the test due to blockage effects caused by the ice accretion. This was accounted for, and analysis of it is shown in a later section.

\section{Mesh Creation}

The commercial meshing software, ANSYS ICEM, was used to produce the mesh. This package was chosen due its ability to implement easy to write macro scripts, allowing the meshing process to be automated for all required re-meshing steps. It should be noted that an alternative meshing software may be required if moving onto whole engine geometries.

In its current form, ICICLE is a 2D code, so a two-dimensional CFD domain was required. Due ICEM's limited 2D meshing capabilities, a thin 3D domain was found to be the easiest implementation.

Surface elements were produced on the blade surface, onto which prism layers were grown. A mesh density was created around the blade to capture the necessary vortical structures in this region. The mesh produced on the "clean" stator, prior to accretion is shown in figure 5.

\section{FIGURE 5 Computational mesh of the initial "clean" geometry}

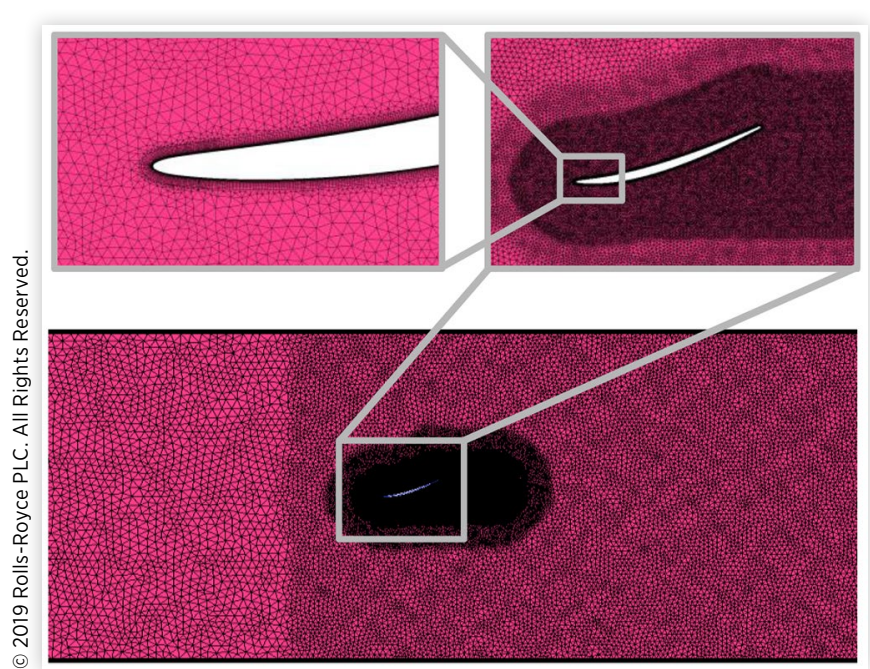




\section{Flow Field Update Methodology}

\section{Flow Field Solution}

The flow solution was calculated using the commercial CFD software, ANSYS FLUENT. Periodic boundary conditions were applied to prevent end wall effects in the spanwise direction. Inlet and outlet pressure boundary conditions were set to match the freestream velocities to the experimental data. The flow solution was solved using a three-dimensional RANS solver, using the $\mathrm{k} \omega$-SST turbulence model, with second order discretisation. Due to the experiments operating up to Mach 0.4, a compressible solver was used.

For the first few time-steps convergence was assumed once the flow residuals were below $1 \times 10^{-4}$. On the latter time-steps the non-smooth accretion geometry led to large pressure surface separations. This caused unsteady wake vortex shedding in the experiment. The RANS calculations struggled to resolve these unsteady flow structures and as a result, the flow downstream of the stator was not reliable, and also caused poor residual convergence.

To determine if URANS simulations were necessary for the geometries tested, a comparison was made. Experimental video data was analysed to determine a representative accretion profile to be studied (taken after 90 seconds of accretion). A RANS and URANS simulation was completed using the same geometry and boundary conditions. The unsteady wake from the iced stator is shown in figure 7, as computed in the URANS calculation. An average pressure coefficient $\left(\mathrm{C}_{\mathrm{p}}\right)$ distribution over the accretion and stator suction surface was calculated from the URANS data, and compared to RANS data, as shown in figure 7. The transparent red curves show the $\mathrm{C}_{\mathrm{p}}$ distributions for each time-step computed, and the dark red line is the time averaged $C_{p}$ distribution. The error bars show one standard deviation in $\mathrm{C}_{\mathrm{p}}$ for all computed time steps. The data showed that on the RANS simulation was nearly within one standard deviation from the URANS for the pressure surface, however much greater difference

\section{FIGURE 6 Contours of normalised vorticity magnitude of} the transient simulation of an iced stator blade after $90 \mathrm{~s}$ of accretion, showing the convection of the trailing edge vortex vortex

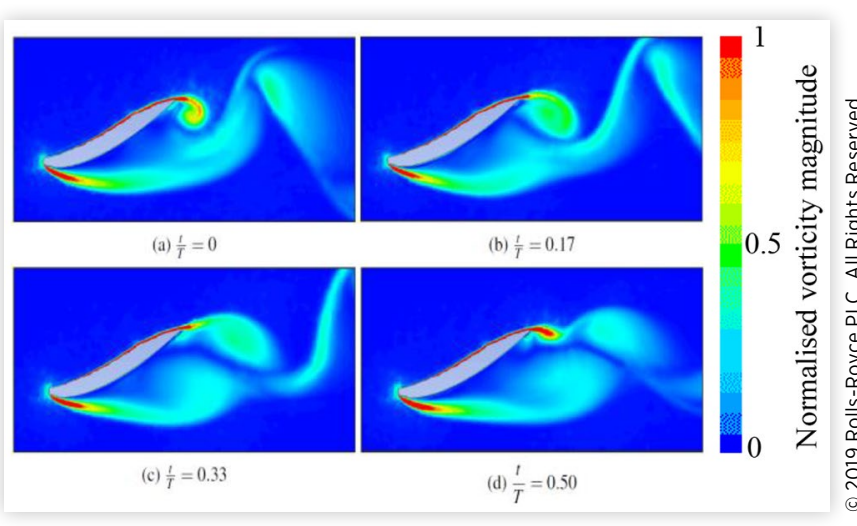

FIGURE 7 Pressure coefficient distribution over the iced stator (shown in figure 6) for the RANS and URANS simulation

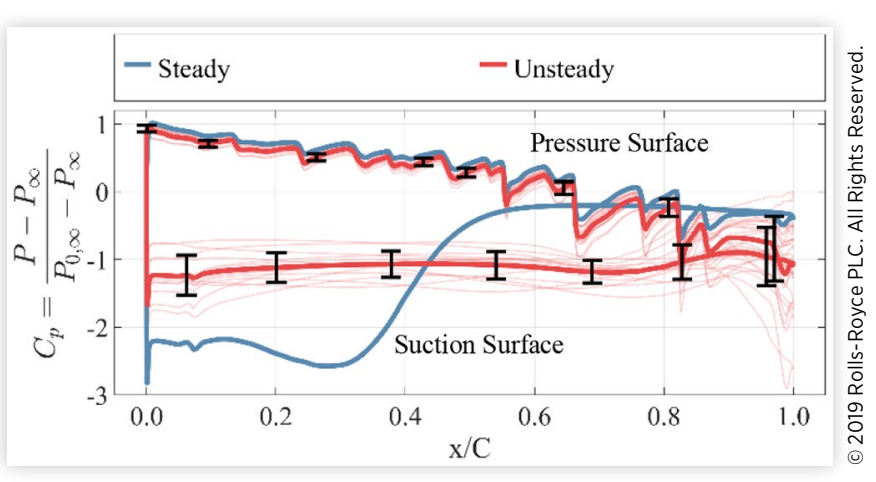

occurred on the suction surface. For determining ice growth, which primarily occurs on the pressure surface, it was deemed unnecessary to resolve the flow structures on the suction surface. Because of these findings, it was concluded to use a RANS solver, however it was noted that the particle trajectory calculations downstream of the stator would likely be incorrect.

\section{Mesh Dependency Study}

A mesh dependency study was carried out to determine the mesh size required for the computational domain. In order to quantify the dependence of the flow solution on the mesh size, a surface at a wall distance of $5 \mathrm{~mm}$ from the stator was produced. On this surface, total pressure, static pressure and velocity $\left(\mathrm{P}_{0}, \mathrm{P}, \mathrm{V}\right)$ were mass averaged. The results of this study is shown in figure 8 , and from this it was concluded that the element size used for the case with 4.5 million elements would be used for the ongoing simulations. With these parameters, a maximum $\mathrm{y}^{+}$of 1 was computed on the stator surface.

\section{Top Level Loop}

The automated re-meshing and flow solution process was controlled by a top-level script created in MATLAB. This allowed for the data to be transferred between ICICLE, meshing in ICEM and CFD in FLUENT.

FIGURE 8 Variation of mass averaged flow parameters $(X)$ at a location $5 \mathrm{~mm}$ from the stator pressure surface, normalised by the value from the simulation with maximum cell count $\left(\mathrm{X}_{0}\right)$

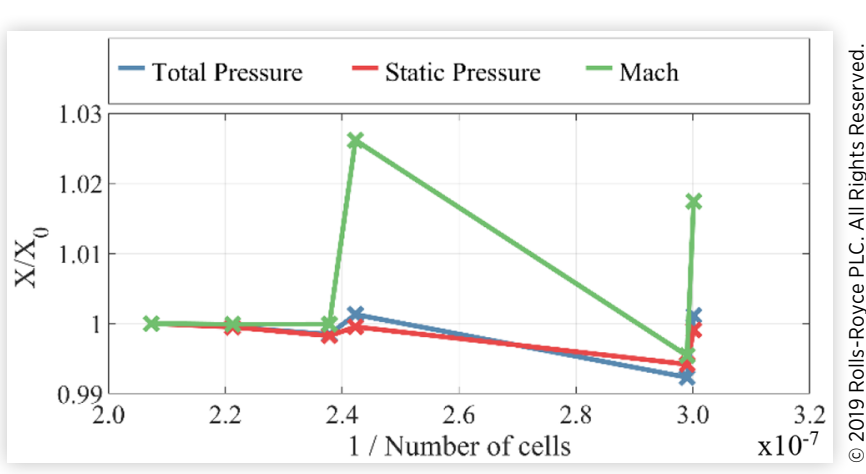

(c) 2019 Rolls-Royce PLC. All Rights Reserved. 
After the first time-step, the smoothed accretion geometry was passed into ICEM, and a spline was fit to the accretion profile. The same meshing parameters were used for all timesteps, but the density around the stator moved accordingly to accommodate the change in accretion size. The mesh was then transferred into FLUENT, a converged flow solution was obtained, and then an axial plane of data was exported to be used within ICICLE.

\section{Blockage Effects}

As accretion built up on the stator blade, blockage effects led to a reduction in Mach number over the test time (the rig maintained constant exit static pressure and constant total pressure at the inlet to the bellmouth). To determine the variation of flow parameters at the main CFD domain inlet, video data from the experiment was used to determine representative ice profiles at a range of blockages during a representative test. These accretion profiles were then used in a CFD simulation of the whole domain to determine the conditions at the plane associated with the inlet of the main CFD domain.

The blockage was quantified by the square root of the volume of the total body (accretion + stator) multiplied by the frontal area of the total body, normalized by the value prior to test (stator only) - equation 1. This parameter was chosen because the frontal area is the most dominant parameter, but ice can grow in a direction which would not be detected by the frontal area or plan area (figure 9) and will affect the loss over the stator.

$$
B=\left.\sqrt{\operatorname{vol} \times A_{\text {front }}}\right|_{\text {accretion }+ \text { stator }} /\left.\sqrt{v o l \times A_{\text {front }}}\right|_{\text {stator }}
$$

Figure 10 shows mass averaged variation in total pressure, static pressure, temperature and Mach number at the inlet plane of the main CFD domain for a range of blockages. The profiles of each variable across the plane were interpolated to determine the inlet profile to be used in the simulation for the specified blockage. These simulations at a range of blockage levels were used within ICICLE, to determine the particle state (melt ratio, particle diameter, aspect ratio, temperature etc.) at the inlet to the main CFD domain. These states were

FIGURE 9 Accretion profile not detected by the frontal area or plan area area

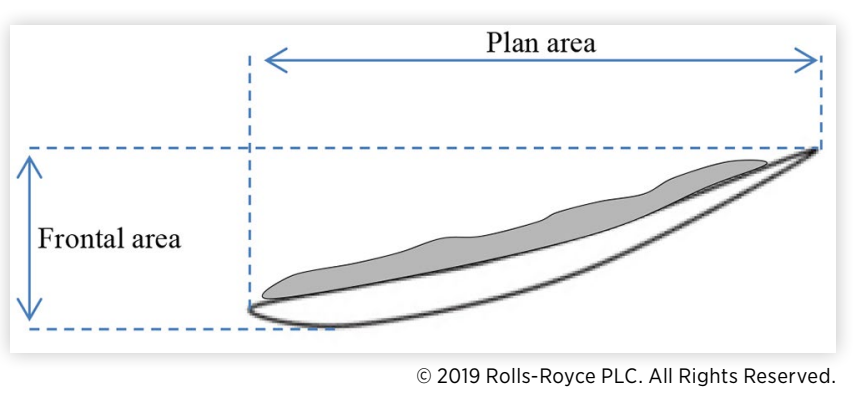

(c) 2019 Rolls-Royce PLC. All Rights Reserved.
FIGURE 10 Variation in mass averaged flow parameters with blockage at the main CFD domain inlet normalised by the values at $B=1$.

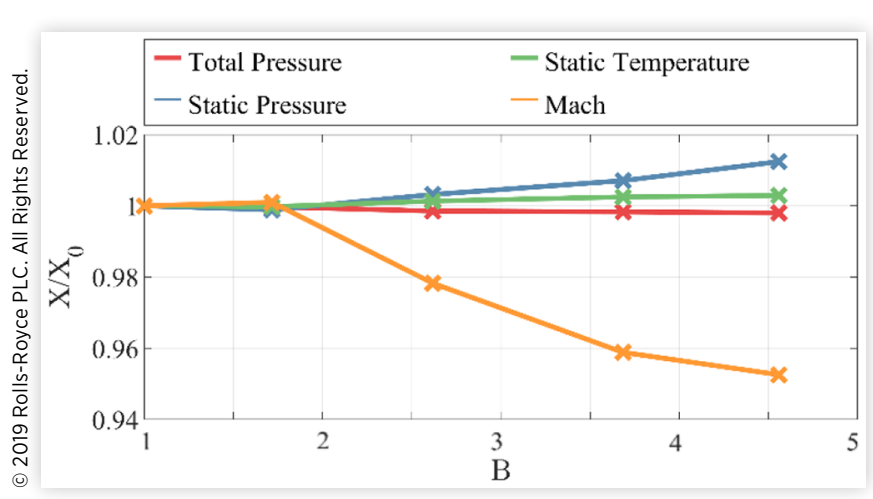

interpolated to determine the particle inlet conditions for the specified blockage. This process would not be required if the whole CFD domain was computed on each time-step but was implemented to aid the required computational time.

The variation in Mach number with blockage, shown in figure 10 indicates the necessity to update the flow field as the accretion grows. If there is minimal slip between the particle and freestream velocity, the reduced velocity with blockage (for a constant operating point) leads to an increase in natural melt of the particle. Previous research found that the variation of sticking efficiency with particle melt ratio changes dramatically either side of the "plateau region"[7] and therefore a small change in melt ratio can have a large effect on the generated accretion.

\section{Simulations}

Four different types of simulations were carried out for the analysis in this paper.

1. A baseline study was computed with no flow field updating. This simulation will be referenced as "no update".

2. The geometry of the body in the simulation was updated at discrete time-steps (of 15 seconds) according to the numerically predicted accretion growth. The new geometry was used to determine new particle impact calculations, but the new flowfield was not computed. This method will be referenced as "geometry update".

3. This simulation type updated the geometry and adjusted the particle state at the inlet based on the blockage, and will be referenced in this paper as "geometry + state update". This was used to de-couple the effects of particle trajectory changes caused by the updated flow field with the effect of the particle state.

4. Simulations were computed with full flow field and geometry updating at discrete time-step intervals (15 seconds), and will be referenced as "flowfield update". 
FIGURE 11 Updated body outline for geometry update without flow field update

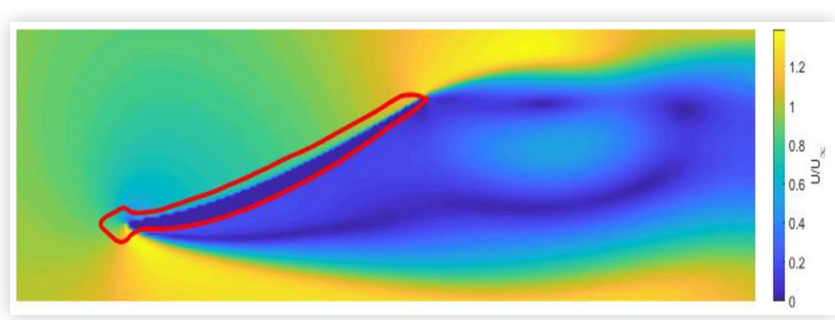

(c) 2019 Rolls-Royce PLC. All Rights Reserved.

By updating the angle between the surface and incoming particles in the "geometry update" method, the stick/bounce calculations were altered and therefore the overall growth rates changed accordingly. By updating the accretion profile, the particle impact locations were also adjusted due to shadowing effects of the bulging accretion on the leading edge. As the flow field was not updated, the particles trajectories did not deflect around high-pressure regions caused by the growing leading-edge accretion. This is shown in figure 11, where the body outline had propagated out of the boundary layer flow. As a result, the velocity at impact was too high, which will have overestimated the breakup rate. Compared to other particle flow disciplines, ice crystal icing has high Stokes numbers, and as a result the crystals are unlikely to decelerate dramatically in the boundary layer, so this effect may not be too dominant.

Each successive method has an increased computational demand, but has increased levels of fidelity which is expected to increase the solution accuracy. Geometry update can be a very efficient method if the trajectory history is stored, such that the new impact location can be computed based on the new geometry (possible due to the "frozen" flowfield) without re-computing the particle trajectories. State update involves interpolation of state variables, but does not add excessive computational demand. Flowfield update requires a large increase in computational demand (meshing, flow solution, trajectory calculations), and therefore should only be used in conditions for which it is necessary.

\section{Accretion Profiles}

Figures 12-17 show the accretion profiles predicted at each timstep for the numerical simulations and experimental data, over a range of 90 seconds. After 15 seconds the accretion size was sufficiently small that there was little change in blockage, nor a change in surrounding flow field, and as a result all three simulation types collapsed onto a single profile. Numerical simulations had good agreement with the experimental data.

In latter timesteps, for the no update simulation the collection efficiency did not change causing the shape of accretion to also not vary during the simulation, and instead grew in volume.

A large change in accretion profile was observed when the geometry was updated on successive time-steps. In the leading edge region, the particles had increased erosional
FIGURE 12 Numerical and experimental accretion profiles at $\mathrm{t}=15 \mathrm{~s}$

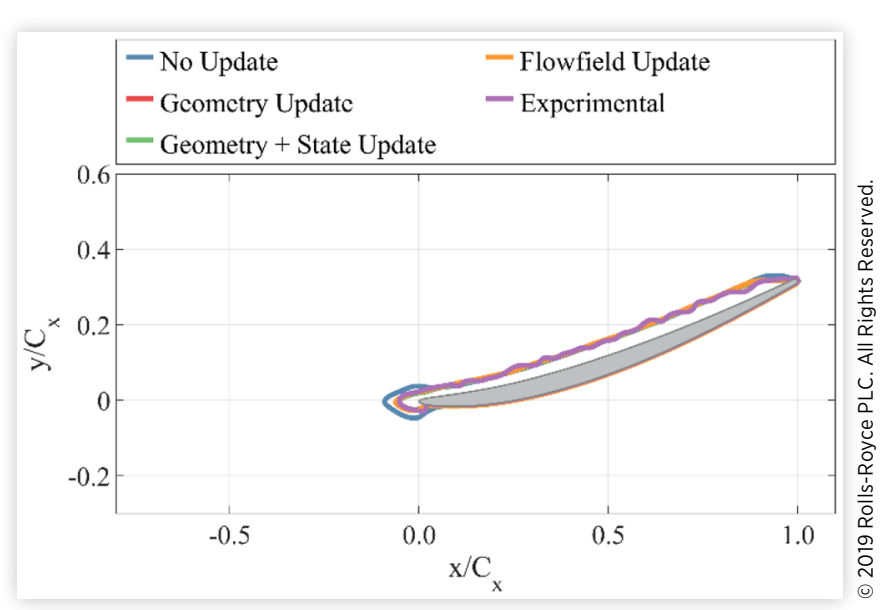

FIGURE 13 Numerical and experimental accretion profiles at $\mathrm{t}=30 \mathrm{~s}$

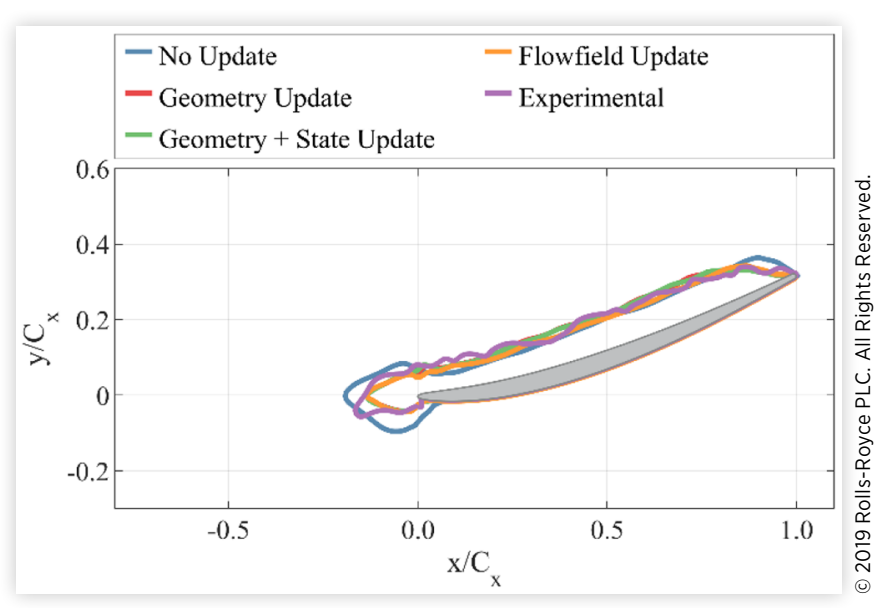

FIGURE 14 Numerical and experimental accretion profiles at $\mathrm{t}=45 \mathrm{~s}$

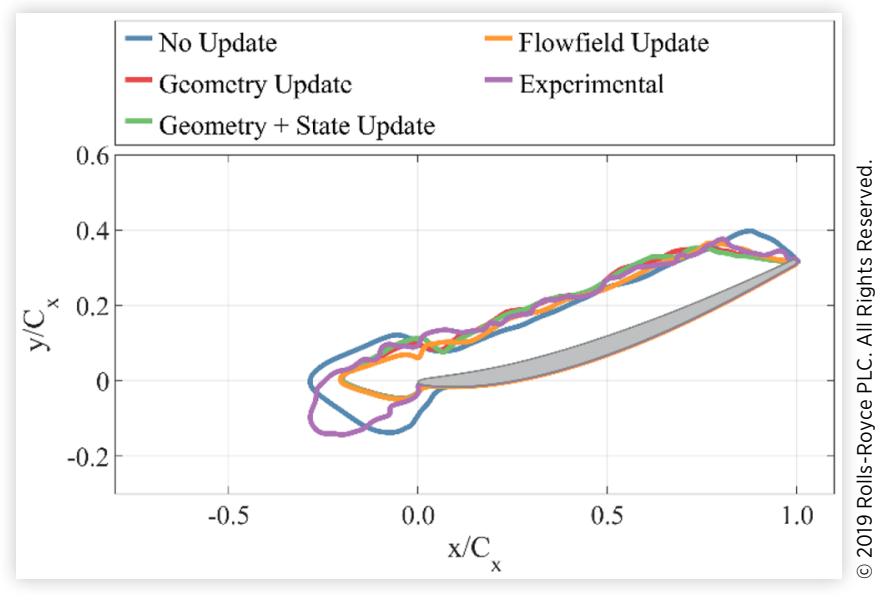

(c) 2019 Rolls-Royce PLC. All Rights Reserved. 
FIGURE 15 Numerical and experimental accretion profiles at $\mathrm{t}=60 \mathrm{~s}$

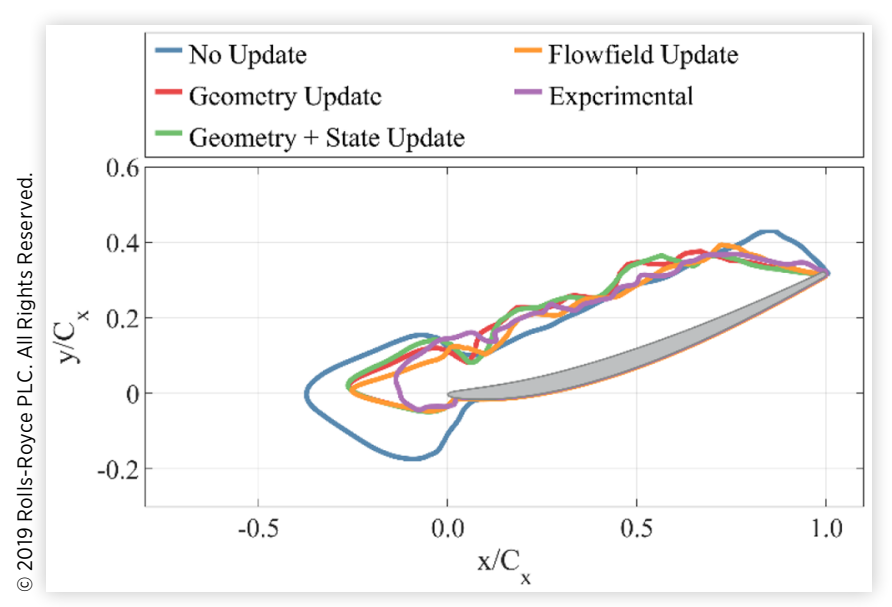

FIGURE 16 Numerical and experimental accretion profiles at $\mathrm{t}=75 \mathrm{~s}$

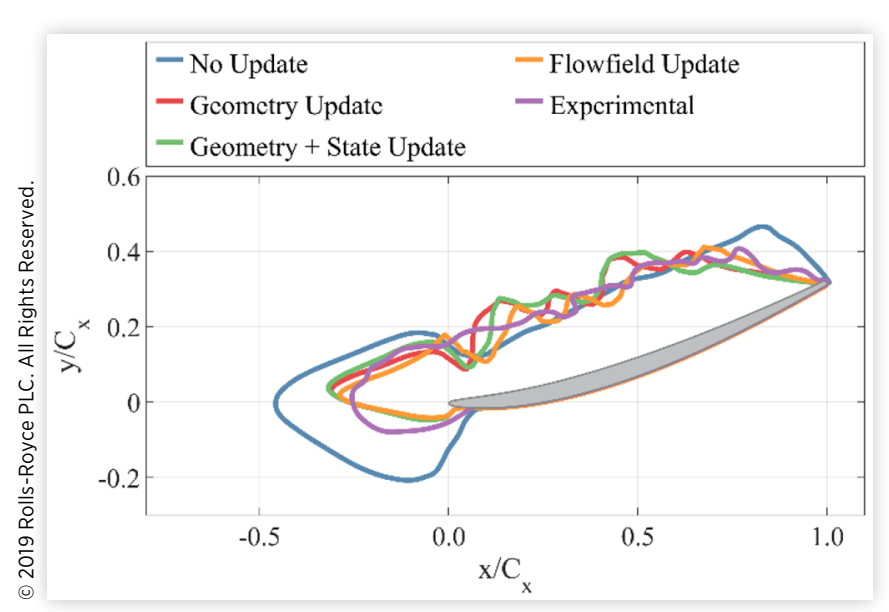

FIGURE 17 Numerical and experimental accretion profiles at $\mathrm{t}=90 \mathrm{~s}$

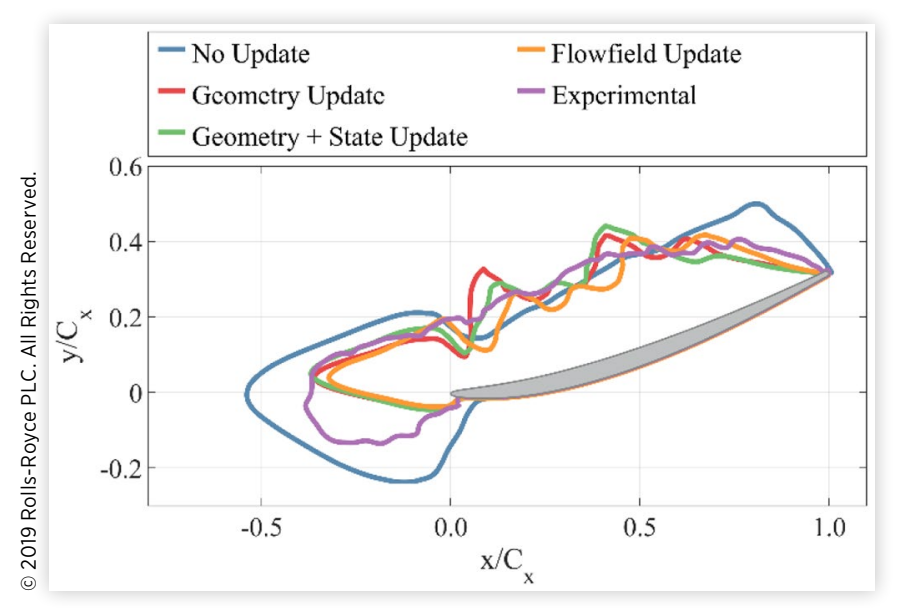

(c) 2019 Rolls-Royce PLC. All Rights Reserved. effect and decreased sticking efficiency. This decreased the local growth rate and prevented the unrealistic leading edge bulge created in the no update solution. Further changes also occurred on the pressure surface. It is thought that this was primarily caused by the change in surface angle affecting the impact behavior, but also caused by shadowing effects of the leading edge accretion on latter timesteps. Good agreement was observed between the experimental and numerical data on the pressure surface, with a significant improvement over the no update simulations. The key difference being at the trailing edge of the pressure surface, in which the decreased accretion rate was not predicted with the no update solution.

When the particle state was adjusted based on the blockage, only minor differences in accretion profile was observed. This suggests that the change in particle state (melt ratio, particle size, aspect ratio etc.) with a changing flow blockage in the range studied here is insignificant. It is expected that this effect will be more dominant when cascade tests are performed, due to the accretion creating a significantly higher blockage.

With flow field updating, little changes in accretion profile were observed compared to geometry updating for $\mathrm{t}<60 \mathrm{~s}$. Between 60 and $90 \mathrm{~s}$, the leading edge accretion differed. With flow field updating, the height of the leading edge accretion was smaller than for geometry updating. This was caused by the large accretion creating an enlarged stagnation region, deflecting the flow streamlines in the local area.

The key difference between the numerically predicted profiles with at least geometry update (simulation type (2)-(4)) compared to the experimental data was at the suction surface leading edge. In the experiment, due to the high relative humidity (45\%), the leading edge accretion was relatively wet and hence had a low stiffness. This led to the leading edge accretion bending downwards under the aerodynamic load. This is not accounted for in the current version of ICICLE, and hence explains the difference in predicted accretion profiles in this region. It should be noted that between 45 and 60 seconds, a leading edge shed occurred, causing the change in leading edge accretion profile. This also cannot be predicted within ICICLE, but it is believed that the bending of the accretion would need to be predicted first, before a shedding model could work accurately.

For the conditions chosen for this study, the results showed that the change in geometry was more dominant on the accretion profile compared to an updated flow solution. Now the numerical tools have been produced, a range of conditions are to be tested to determine conditions for which flowfield updating is more dominant, and other conditions for which geometry updating is sufficient. Greater levels of blockage will also be further studied (such as in a cascade) to see if only the particle state needs updated for geometries in which the accretion fills a greater proportion of the flow path. However it is expected that at these high blockage ratios, the particle trajectories will be heavily dominated by the flowfield, and a full flowfield update may be required. For all conditions, the dependency on the timestep between updates will be studied to find optimal values. 


\section{Growth Rates}

Figure 18 shows the predicted growth rates for each simulation type, alongside the experimental data. With no flow field or geometry update a nonlinear growth rate initially occurred as the blade temperature decreased and ice started to form. Once an ice layer had stabilized ( $>30 \mathrm{~s})$, the growth rate remained approximately constant. With geometry updating the growth rate was lower than the baseline simulation and remained roughly constant for the first 40 seconds and then started to decrease. With flow field updating the growth rate most accurately matched the experimental data. As previously mentioned, a shed event occurred between 45 and 60 seconds, which was not numerically predicted, explaining the difference in volume after this time. The decrease in growth rate with the updated simulations more closely match the experimental data, and it is expected to be caused by the increase in erosional effect as the accretion grows.

\section{Collection Efficiency}

The average collection efficiency of each panel in the computational domain was calculated at the first time step and at the final time-step of the flow field update simulation. Collection efficiency was defined as the fraction of the particles collected by the local body relative to the number injected. Figure 19 shows the results of this study. If no geometry or flow field update was included in the model then the collection efficiency would have remained constant over the simulation. The peak collection efficiency of the clean stator occurred at the leading edge, which was the cause of the leading edge bulge which formed in the baseline simulations. As the accretion grew, each panel collected a different percentage of incoming particles due to the change in incoming particle velocity, relative angle between impact velocity and adjacent surface, and also change in melt ratio. On the latter time steps a lower proportion of particles were collected by the stator, which can also be seen by the reduced growth rate in figure 18 .

\section{FIGURE 18 Comparison of numerical and experimental} growth rates, normalized by the experimental value at 90 seconds

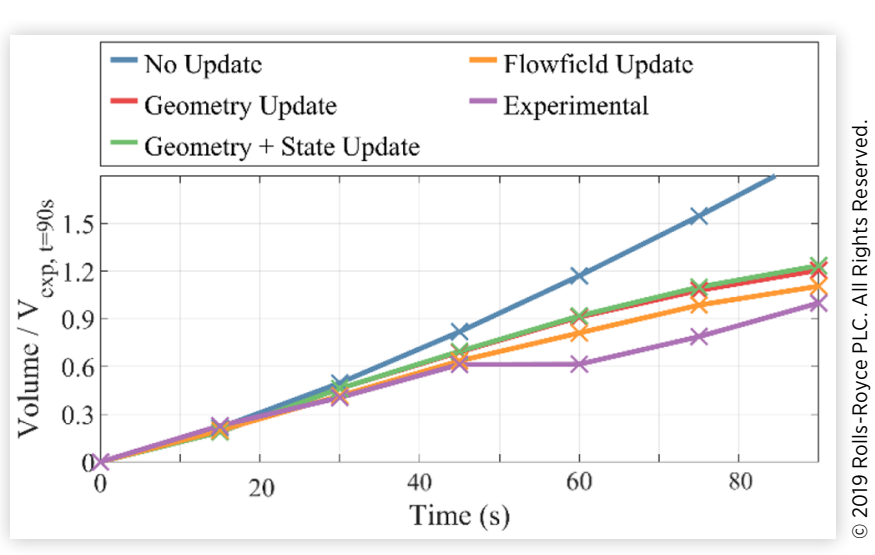

FIGURE 19 Distribution of collection efficiency over the stator for the initial "clean" geometry and final accretion profile predicted after 120 seconds.

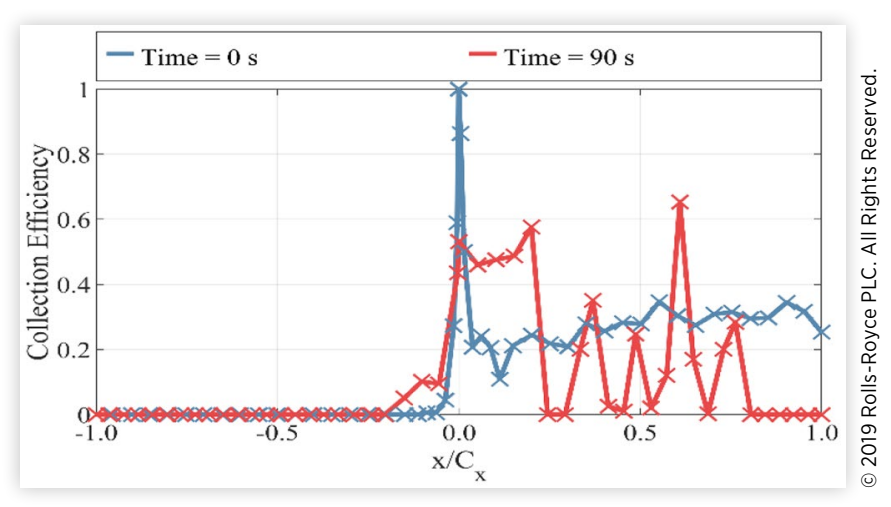

\section{Conclusions}

A fully automated two way coupling between the numerically predicted ice accretion and geometry for trajectory calculations/flowfield has been produced. The work showed that the ice crystal icing accretion profiles predicted by numerical codes is highly dependent on the geometry and flow solution. As an accretion forms, the local surface angle of the body relative to the incoming particle streams changes, affecting the post impact behavior (stick/bounce/shatter). For the conditions studied in this paper, the change in geometry had the most dominant effect on the final accretion profile. By updating the flow solution, in the latter stages of the calculation $(t>60 s)$ a change in accretion profile started to occur relative to only adjusting the geometry. The change in geometry effected the location and size of stagnation region of the stator, leading to a vastly different upstream pressure distribution. The change in pressure forces then altered the particle trajectories, hence altering the final accretion profile. For the high particle velocities studied $(M=0.4)$, their ballistic nature meant that they were less effected by the change in flow solution than was previously expected.

The effect of flow field updating is expected to be highly dependent on the particle size distribution. For large particles, which have high Stokes numbers, they will act balistically and hence be less effected by the change in pressure distribution. As previously noted, for small accretions, the final ice profile is less effected by an updated flow field. For a particle size distribution with a large $D_{v 50}$ and $D_{v 90}$, the particles will behave balistically and are highly erosive - leading to a small overall accretion. In these conditions it is expected that it is less critical to update the flow field. Further work will now go into producing a map of conditions (melt ratio, particle size distribution, and kinetic energy) for which flow field updating is necessary, and others for which the prediction will be sufficiently accurate by only updating the geometry. Finally, work will also go into determining the effect of the time-step between flow field updates, to find an optimum value for a given set of conditions. The work will be applied into a cascade geometry, for which it is expected that large blockage ratios will occur, leading to gross changes in the flowfield and hence trajectory paths, as found in engine icing tests. 


\section{References}

1. Mason, J., Strapp, W., and Chow, P., “The Ice Particle Threat to Engines in Flight," in 44th AIAA Aerospace Sciences Meeting and Exhibit, 2006.

2. Bucknell, A., Mcgilvray, M., Gillespie, D.R.H., Jones, G., and Reed, A., "ICICLE: A Model for Glaciated \& Mixed Phase Icing for Application to Aircraft Engines," in SAE Int. Conf. Icing Aircraft, Engines Struct., Minneapolis, MN, USA, June 17-21.

3. Bucknell, A., Mcgilvray, M., Gillespie, D., Jones, G., and Collier, B., "A Three-Layer Thermodynamic Model for Ice Crystal Accretion in Gas Turbine Engines," in Submitt. SAE Int. Conf. Icing Aircraft, Engines Struct., Minneapolis, MN, USA, June 17-21, 2019.

4. Villedieu, P., Trontin, P., and Chauvin, R., "Glaciated and Mixed-Phase Ice Accretion Modeling Using ONERA 2D Icing Suite," Trans. Japanese Soc. Med. Biol. Eng. 51(SUPPL):1-34, 2013.

5. Wright, W., Jorgenson, P., and Veres, J., "Mixed Phase Modeling in GlennICE with Application to Engine Icing," AIAA Atmospheric and Space Environments Conference, 2010.

6. Habashi, W. and Nilamdeen, S., "Multiphase Approach toward Simulating Ice Crystal Ingestion in Jet Engines," J. Propuls. Power 27(5):959-969, 2011.

7. Bucknell, A., Mcgilvray, M., Gillespie, D.R.H., Jones, G., and Reed, A., "Experimental Studies of Ice Crystal Accretion on an Axisymmetric Body at Engine-Realistic Conditions," in 2018 Atmos. Sp. Environ. Conf. AIAA Aviat. Forum (AIAA 2018-4223), 2018.

8. Bucknell, A., Mcgilvray, M., Gillespie, D., Jones, G., and Collier, B., "A Three-Layer Thermodynamic Model for Ice Crystal Accretion in Gas Turbine Engines," in SAE Int. Conf. Icing Aircraft, Engines Struct., Minneapolis, MN, USA, June 17-21, 2019.

9. Veres, J.P., Jorgenson, P., and Jones, S.M., "Modeling of Highly Instrumented Honeywell Turbofan Engine Tested with Ice Crystal Ingestion in the NASA Propulsion System Laboratory," in 8th AIAA Atmos. Sp. Environ. Conf., 1-20, 2016.
10. Bucknell, A., Mcgilvray, M., Gillespie, D.R.H., Jones, G. et al., "Experimental Study and Analysis of Ice Crystal Accretion on a Gas Turbine Compressor Stator Vane," in SAE Int. Conf. Icing Aircraft, Engines Struct., Minneapolis, MN, USA, June 17-21, 2019, Submitt.

11. El-Batsh, H., "Modeling Particle Deposition on Compressor and Turbine Blade Surfaces Modeling Particle Deposition on Compressor and Turbine Blade Surfaces," 2001.

12. Hedde, T. and Gufford, D., "ONERA Three-Dimensional Icing Model,” AIAA, Jan. 1995.

13. Forsyth, P., Gillespie, D.R.H., and Mcgilvray, M., "Development and Applications of a Coupled Particle Deposition Dynamic Mesh Morphing Approach for the Numerical Simulation of Gas Turbine Flows," in ASME Turbo Expo 2017, 2017, 1-15.

14. Trontin, P., Blanchard, G., Kontogiannis, A., and Villedieu, P., "Description and Assessment of the New ONERA 2D Icing Suite IGLOO2D," in 9th AIAA Atmos. Sp. Environ. Conf. AIAA Aviat. Forum (AIAA 2017-3417), 2017.

15. Baumert, A., Bansmer, S., Trontin, P., and Villedieu, P., "Experimental and Numerical Investigations on Aircraft Icing at Mixed Phase Conditions," Int. J. Heat Mass Transf. 123:957-978, 2018.

\section{Acknowledgements}

The authors would like to thank Rolls-Royce Plc for funding the research; the UK Engineering and Physical Sciences Research Council (EPSRC) for the CDT in Gas Turbine Aerodynamics studentship (grant ref. EP/L015943/1).

\section{Contact Information}

\author{
Jonathan Connolly \\ Oxford Thermofluids Institute \\ Jonathan.connolly@eng.ox.ac.uk
}

\section{Definitions/Abbreviations}

ICICLE - Ice Crystal Icing ComputationaL Environment 